\title{
A Division Algebra for Sequences Defined on all the Integers
}

\author{
By D. H. Moore
}

The convolution ring, $s$, of sequences defined on the nonnegative integers, and the embedding of this ring in a field, have been discussed by Brand [1], Moore [2], [3], Traub [6], and others. Brand [1] specifically mentions that the field in which he embeds $S$ is a field of ordered pairs of members of $S$. Traub does not identify his field and does not mention "ordered pairs", but he mentions an analogy to Mikusinski's work [7], and so he probably had in mind the same field of ordered pairs as did Brand. In [2] this writer showed that it was not necessary to create such a field of ordered pairs since there already existed a more natural, less abstract field in which to embed $S$. It is the purpose of this article to introduce this already existing and more natural field, $\mathcal{F}$, in which $S$ may be embedded.

It will be assumed that the reader is familiar with the convolution algebra of sequences as given in [1], [3], and [6] to the point of recognizing $S$ as an integral domain in which convolution products defined by

$$
\left\{a_{\nu}\right\}\left\{b_{\nu}\right\}=\left\{\sum_{\mu=0}^{\nu} a_{\mu} b_{\nu-\mu}\right\}
$$

contain no divisors of zero, in which the multiplicative unity is the sequence

$$
\{1,0,0,0, \cdots, 0, \cdots\} \text {, }
$$

in which sequences of the form

$$
\{c, 0,0,0, \cdots, 0, \cdots\}
$$

behave like numbers and are identified with numbers:

$$
c=\{c, 0,0,0, \cdots, 0, \cdots\}
$$

in which the sequence

$$
\{0,1,0,0,0, \cdots, 0, \cdots\}
$$

is a shift operator denoted by " $\tau$ ", in which the sequence

$$
\{1,1,1, \cdots, 1, \cdots\}
$$

is a summing operator denoted by " $\sigma$ ", and in which members of $\delta$ have operational forms in terms of $\tau$ and/or $\sigma$.

The sequences $\sigma$ and $\tau$ are related by the equation

$$
\sigma(1-\tau)=1
$$

and since $S$ has no divisors of zero we introduce fractions and write (for example)

$$
\begin{aligned}
& \sigma=\frac{1}{1-\tau}, \\
& \frac{1}{\sigma}=1-\tau=\{1,-1,0,0,0, \cdots\} .
\end{aligned}
$$

Received July 13, 1965. Revised August 19, 1965. 
The fraction $1 / \tau$, for example, does not exist as a member of $\mathcal{S}$. But $1 / \tau$ will exist as a member of $\mathcal{F}$.

Let $\mathcal{F}$ be the class of number valued sequences defined over the integral domain, $J$, each of which assigns at most a finite number of nonzero values to negative integers. For each member of $\mathcal{F}$ there is a least integer, $m$, to which the sequence assigns a nonzero value; the sequence will be said to enter at $m$, and the members of $\mathcal{F}$ will be called entering sequences. Equality, sums, and products with numbers, of members of $\mathcal{F}$ are defined in the usual termwise way. A suggested notation for such a sequence is

$$
\{1,2,3 \mid 4,5,6, \cdots\}
$$

where the vertical line-playing a role like a decimal point-separates values assigned to the negative integers from values assigned to the nonnegative integers, and the zeros assigned on the left are omitted for convenience.

Let $\nu$ be a variable on $J$. We define the unit step formula " $u(\nu)$ ":

$$
u(\nu)= \begin{cases}0, & \nu<0, \\ 1, & \nu \geqq 0 .\end{cases}
$$

Then $\{u(\nu)\}$ and $\{(\nu+1) u(\nu)\}$ (for example) are members of $\mathcal{F}$, whereas $\{\nu+1\}$ is not. The braces serve to bind out " $\nu$ " converting a formula into a notation for a sequence.

There is a natural one-to-one correspondence between $\mathcal{S}$ (sequences defined on the nonnegative integers) and the subclass, $\mathcal{F}_{0}$, of $\mathcal{F}$ consisting of sequences which enter at nonnegative points:

$$
\left\{a_{0}, a_{1}, a_{2}, \cdots\right\} \leftrightarrow\left\{\cdots, 0,0 \mid a_{0}, a_{1}, a_{2}, \cdots\right\} .
$$

The convolution, or convolution product, of two sequences $a$ and $b$ from $F$ is defined by

$$
a b=\left\{\sum_{\mu=-\infty}^{+\infty} a_{\mu} b_{\nu-\mu}\right\} .
$$

If $c$ enters at $\alpha$ or to the right of $\alpha$ and $b$ enters at $\beta$ or to the right of $\beta$, then

$$
\begin{gathered}
(a b)_{\nu}= \begin{cases}\sum_{\mu=\alpha}^{\nu-\beta} a_{\mu} b_{\nu-\mu}, & \nu \geqq \alpha+\beta, \\
0, & \nu<\alpha+\beta,\end{cases} \\
a b=\left\{u(\nu-\alpha-\beta) \sum_{\mu=\alpha}^{\nu-\beta} a_{\mu} b_{\nu-\mu}\right\} .
\end{gathered}
$$

The summation limits are finite in (5) since the sequences are entering sequences. In particular, if $a$ and $b$ are members of the subclass $\mathcal{F}_{0}$, we may take $\alpha=\beta=0$ in (5) and (5) becomes

$$
a b=\left\{u(\nu) \sum_{\mu=0}^{\nu} a_{\mu} b_{\nu-\mu}\right\} .
$$

A comparison of (1) and (6) shows that the correspondence (2) is an isomorphism under convolution; we embed $S$ in $F$, identify $S$ with $F_{0}$, elevate (2) to an 
equality, and permit any notation for a member of $s$ to be used as a notation for the corresponding member of $\mathcal{F}_{0}$. In particular

$$
\begin{aligned}
1 & =\{\cdots, 0,0,0 \mid 1,0,0,0, \cdots, 0, \cdots\} \\
\sigma & =\{\cdots, 0,0,0 \mid 1,1,1, \cdots, 1, \cdots\} \\
\tau & =\{\cdots, 0,0,0 \mid 0,1,0,0,0, \cdots, 0, \cdots\} \quad m=\text { positive integer. } \\
\tau^{m} & =\{\cdots, 0,0,0 \mid \underbrace{0,0, \cdots, 0}_{m \text { zeros }}, 1,0,0,0, \cdots\}
\end{aligned}
$$

Defining $\zeta$ by :

$$
\zeta=\{1 \mid 0,0,0, \cdots, 0, \cdots\}
$$

we have

$$
\zeta^{m}=\underbrace{\{1,0,0,0, \cdots, 0}_{m \text { digits }} \mid 0,0,0, \cdots, 0, \cdots\} \quad m=\text { positive integer. }
$$

Equations (7) and (9) may be verified by induction. Using (5) we may verify that

$$
\begin{aligned}
\tau \zeta & =1 \\
\tau^{m+n} & =\zeta^{m} \tau^{n} \\
\zeta^{m+n} & =\zeta^{m} \zeta^{n} \\
\tau^{m}\left\{a_{v}\right\} & =\left\{a_{v-m}\right\} \\
\zeta^{m}\left\{a_{v}\right\} & =\left\{a_{v+m}\right\}
\end{aligned}
$$

$m, n$ positive integers.

Under ordinary addition and convolution multiplication $\mathcal{F}$ is a field. We need only verify here that each nonzero member of $\mathcal{F}$ has a multiplicative inverse. To begin with, every sequence of the form

$$
\left\{a_{0}, a_{1}, a_{2}, \cdots\right\}
$$

in which $a_{0} \neq 0$ (the sequence enters at the origin) has an inverse:

$$
\left\{x_{0}, x_{1}, x_{2}, \cdots\right\}
$$

which may be evaluated as follows:

$$
\begin{aligned}
\left\{a_{0}, a_{1}, a_{2}, \cdots\right\}\left\{x_{0}, x_{1}, x_{2}, \cdots\right\} & =\{1,0,0,0, \cdots\} \\
a_{0} x_{0} & =1 \\
a_{0} x_{1}+a_{1} x_{0} & =0 \\
a_{0} x_{2}+a_{1} x_{1}+a_{2} x_{0} & =0
\end{aligned}
$$

Since the only division involved in solving for the $x$ 's is division by $a_{0}$, and $a_{0} \neq 0$, the $x$ 's exist and so the desired inverse exists.

Finally, let $a$ be any nonzero member of $\mathcal{F}$ which does not enter at the origin. 
Since $a$ is an entering sequence, there exists a sequence $A$ and a positive integer $m$ such that either

$$
a=\tau^{m} A \text { or } \quad a=\zeta^{m} A
$$

where $A$ enters at the origin, and so has an inverse $A^{-1}$ by the preceding paragraph. Then either

$$
\left(A^{-1} \zeta^{m}\right) a=1 \text { or }\left(A^{-1} \tau^{m}\right) a=1
$$

and so, in any case, $a$ has a multiplicative inverse, and $\mathcal{F}$ is a field.

Since $\mathcal{F}$ contains no divisors of zero, products lead to the introduction of fractions:

$$
\left.\begin{array}{c}
a, b, c \in \mathcal{F} \\
\text { and } \\
a b=c \\
\text { and } \\
a \neq 0
\end{array}\right\} \Rightarrow\left\{\begin{array}{c}
\frac{c}{a} \text { exists as a member of } \mathcal{F} \\
\text { and } \\
\frac{c}{a}=b \\
\text { and } \\
a\left(\frac{c}{a}\right)=c .
\end{array}\right.
$$

In particular

$$
\zeta=\{1 \mid 0,0,0, \cdots\}=\frac{1}{\tau}=\frac{\{1,0,0,0, \cdots\}}{\{0,1,0,0,0, \cdots\}}
$$

and $1 / \tau$ exists as a member of $\mathcal{F}$.

Members of $\mathcal{F}$ may be put into operational form in terms of $\sigma, \tau$, and/or $\zeta$.

Example 1.

$$
\begin{aligned}
\left\{\frac{\nu(\nu-1)}{2} u(\nu+2)\right\} & =\{3,1 \mid 0,0,1,3,6,10,15, \cdots\} \\
& =\zeta^{2}\left\{\frac{(\nu-2)(\nu-3)}{2} u(\nu)\right\}=\frac{\zeta^{2}}{2}\left\{\left(\nu^{2}-5 \nu+6\right) u(\nu)\right\} \\
& =\frac{\zeta^{2}}{2}\left(\sigma^{2} \tau+2 \sigma^{3} \tau^{2}-5 \sigma^{2} \tau+6 \sigma\right)
\end{aligned}
$$

where $\{\nu u(\nu)\}=\sigma^{2} \tau$ and $\left\{\nu^{2} u(\nu)\right\}=\sigma^{2} \tau+2 \sigma^{3} \tau^{2}$ as shown in [2], and as may be checked straightforwardly. Then

$$
\left\{\frac{\nu(\nu-1)}{2} u(\nu+2)\right\}=\sigma^{3}-2 \sigma^{2} \zeta+3 \sigma \zeta^{2}
$$

In Traub [6, p. 196], every quotient of "generalized" sequences with a nonzero denominator equals a shift operator times an ordinary sequence. Thus, in Traub's notation,

$$
\frac{f}{g}=\frac{f}{\omega^{i} e}=\omega^{-i} \frac{f}{e}
$$


where $f / e$ equals an ordinary sequence since $e$ assigns a nonzero value to the origin; $\omega^{-i}$ is a shift operator, and is a "generalized" sequence-an ordered pair of ordinary sequences. In comparison, in the present paper, we are dealing with entering sequences (defined on $J$ ) instead of ordered pairs, and every quotient, $b / a$, of entering sequences (with nonzero denominator) equals an entering sequence. In evaluating $b / a$ we may replace $a$, as in (10), by $\tau^{m} A$ or $\zeta^{m} A$, as appropriate, and obtain respectively

$$
\frac{b}{a}=\zeta^{m} \frac{b}{A} \quad \text { or } \quad \frac{b}{a}=\tau^{m} \frac{b}{A}
$$

where $b / A, \zeta^{m}$, and $\tau^{m}$ are all entering sequences.

Example 2.

$$
\begin{aligned}
\frac{\{1,-1,1,-1,1,-1, \cdots\}}{\{1,1,1 \mid 1,1, \cdots\}} & =\frac{1 /(1+\tau)}{\zeta^{3} \sigma}=\tau^{3} \frac{1-\tau}{1+\tau} \\
& =\tau^{3}\{1,-2,2,-2,2,-2, \cdots\} \\
& =\{0,0,0,1,-2,2,-2,2,-2, \cdots\} .
\end{aligned}
$$

Example 3.

$$
\begin{aligned}
\frac{\{3,1 \mid 0,0,1,3,6,10,15, \cdots\}}{\{0,0,0,1,3,3,1,0,0,0, \cdots\}} & \quad \text { (see example 1) } \\
= & \frac{\sigma^{3}-2 \sigma^{2} \zeta+3 \sigma \zeta^{2}}{\tau^{3}(\tau+1)^{3}} \\
= & \frac{1}{(1-\tau)^{3}}-2 \frac{1}{(1-\tau)^{2}} \frac{1}{\tau}+3 \frac{1}{\tau^{3}(\tau+1)^{3}} \frac{1}{\tau^{2}} \\
= & \left(\frac{6}{\tau^{3}}-\frac{8}{\tau^{4}}+\frac{3}{\tau^{5}}\right) \frac{1}{\left(1-\tau^{2}\right)^{3}} \quad \text { (omitting several algebraic steps) } \\
= & \left(6 \zeta^{3}-8 \zeta^{4}+3 \zeta^{5}\right)\{1,0,3,0,6,0,10,0,15,0, \cdots\} \\
= & \{6,0,18 \mid 0,36,0,60, \cdots\} \\
& +\{-8,0,-24,0 \mid-48,0,-80,0, \cdots\} \\
& +\{3,0,9,0,18 \mid 0,30,0,45, \cdots\} \\
= & \{3,-8,15,-24,36 \mid-48,66,-80,105, \cdots\} .
\end{aligned}
$$

The last result may be checked by cross multiplication:

$$
\begin{aligned}
\{0,0,0,1,3,3,1,0,0,0, \cdots\}\{3,-8,15,-24,36 \mid & -48,66,-80,105, \cdots\} \\
& =\{3,1 \mid 0,0,1,3,6,10,15, \cdots\} .
\end{aligned}
$$

A convenient way to multiply two entering sequences is to ignore the vertical lines at first, and then insert a vertical line in the final answer, following rules similar to those for the insertion of a decimal point in a product of decimals. 
George Boole's operator, $E$, $[4$, p. 16] which shifts a sequence to the left and replaces by zero the terms which pass the origin, operates only on sequences which vanish to the left of the origin:

$$
E^{n}\{f(\nu) u(\nu)\}=\{f(\nu+n) u(\nu)\}, \quad n=\text { nonnegative integer. }
$$

Thus $E$ cannot be identified with $\zeta$; neither is $E$ to be discarded, since there is no convolution product to do the job that $E$ does, and that job is important. However, George Boole's symbolic method [4, p. 215] is salvaged if $E$ is replaced by $\zeta$ as discussed in [2]. Thus, Boole's symbolic equation [4, pp. 217, 218]

$$
\frac{b^{x}}{E-a}=\frac{b^{x}}{b-a}+c a^{x}, \quad c=\text { arbitrary constant } a, b \text { numbers }
$$

becomes:

$$
\frac{\left\{b^{\nu} u(\nu)\right\}}{\zeta-a}=\frac{\left\{b^{\nu} u(\nu)\right\}}{b-a}+\frac{\left\{a^{\nu} u(\nu)\right\}}{a-b} .
$$

This follows from the equation

$$
\left\{c^{\nu} u(\nu)\right\}=\frac{1}{1-c \tau}=\frac{\zeta}{\zeta-c}, \quad c=\text { number }
$$

which is easily checked by cross multiplication. To prove (11) we have

$$
\begin{aligned}
\frac{\left\{b^{\nu} u(\nu)\right\}}{\zeta-a} & =\frac{\zeta}{\zeta-b} \frac{1}{\zeta-a}=\frac{1}{b-a} \frac{\zeta}{\zeta-b}+\frac{1}{a-b} \frac{\zeta}{\zeta-a} \\
& =\frac{\left\{b^{\nu} u(\nu)\right\}}{b-a}+\frac{\left\{a^{\nu} u(\nu)\right\}}{a-b} .
\end{aligned}
$$

When operational forms of sequences are expressed in terms of $\zeta$ they match the $Z$-transforms of sequences as used, for example, by Aseltine [5] (hence the use of " $\zeta$ " for the reciprocal of $\tau$ ). For example [5, p. 259]

$$
\{u(\nu)\}=\sigma=\frac{1}{1-\tau}=\frac{\zeta}{\zeta-1} .
$$

But now $\zeta$ is a sequence and not a variable, a formula in $\zeta$ equals a sequence rather than being a "transform" of it, and the introduction of the $\zeta$-forms requires no theory of convergence of power series. In [2, pp. 140-143] it is shown that results previously obtained using the theory of functions of a complex variable including branch cuts and the theory of residues, may be obtained by purely algebraic methods from the field properties of $\mathcal{F}$.

California State Polytechnic College

Pomona, California

1. LouIs Brand, "A division algebra for sequences and its associated operational calculus," Amer. Math. Monthly, v. 71, 1964, pp. 719-728. MR 29 *5069.

2. D. H. Moore, "Convolution products and quotients of sequences. An operational calculus for sequences and for pulsed-data and digital systems," Dissertation, Univ. of Calif., Los Angeles, 1962.

3. D. H. Moore, "Convolution products and quotients and algebraic derivatives of sequences," Amer. Math. Monthly, v. 69, 1962, pp. 132-138. MR $25 * 402$. 
4. George Boole, Calculus of Finite Differences, 4th ed., Chelsea, New York, 1957. MR 20 * 1124.

5. John Aseltine, Transform Method in Linear System Analysis, McGraw-Hill Electrical and Electronics Engineering Series, McGraw-Hill, New York, 1958. MR 21 *6201.

6. J. F. TraUB, "Generalized sequences with applications to the discrete calculus," Math. of Comp., v. 19, 1965, pp. 177-200.

7. J. Mrkusi Kski, Operational Calculus, International Series of Monographs on Pure and Applied Mathematics, Vol. 8, Pergamon Press, New York, and Panstwowe Wydawnictwo Naukowe, Warsaw, 1959. MR 21 *4333. 